ordenada (mêlée, figura 1-C), o alinhamento (touche, figura 1-E), a formação espontânea (ruck, figura 1-D) ou a

\title{
Biomecânica e Traumatologia no Râguebi
}

Dr. João Diogo Silva ${ }^{1}$, Prof. Dr. António Cruz-Ferreira ${ }^{2}$, Dr. Jorge Laíns ${ }^{3}$, Dr. Diogo Lino Moura ${ }^{4}$ ${ }^{1}$ Interno de Medicina Física e de Reabilitação do Centro de Medicina de Reabilitação da Região Centro Rovisco Pais (CMRRC-RP); ${ }^{2}$ Diretor Clínico da Federação Portuguesa de Râguebi, UCSP Mealhada; ${ }^{3}$ Diretor de Serviço de Medicina Física e de Reabilitação do CMRCC-RP; ${ }^{4}$ Docente da Faculdade de Medicina da Universidade de Coimbra, Assistente Hospitalar de Ortopedia do Centro Hospitalar e Universitário de Coimbra.

\begin{abstract}
RESUMO / ABSTRACT
O râguebi é uma das modalidades coletivas com maior taxa de incidência de lesões por tempo de exposição. Estas lesões decorrem na maioria dos casos de movimentos específicos deste desporto, tais como a placagem, o ruck ou o maul. Importa, por isso, compreender as especificidades da modalidade e a sua biomecânica na associação ao risco e ao tipo de lesão desportiva. Os tipos de lesão mais frequentemente reportadas são as roturas musculotendinosas e as ligamentares nos membros inferiores, no entanto é de destacar a incidência importante de graves lesões da cabeça e pescoço, tais como a concussão cerebral e fraturas da coluna cervical. É essencial reunir dados que permitam caracterizar o risco e a especificidade de cada movimento realizado pelo atleta em campo de modo a elaborar programas mais eficazes, tanto na prevenção de lesões, como na reabilitação de atletas, visando a sua completa recuperação e a redução do risco de recidiva.
\end{abstract}

Rugby is one of the team sports with the highest incidence rate of injuries per exposure time. These mainly occur in specific sport movements such as the tackle, ruck, or maul. Therefore, it is important to understand the sports' specificities and biomechanics and link them with its risk and injury type. The most common reported injuries are musculotendinous and ligament ruptures of the lower limbs, however we highlight the important incidence of severe head and neck injuries, like cerebral concussion and cervical spine fractures. It is essential to gather data that enable us to characterize the risk and specify of each movement performed by the athlete, in order to make more effective programs on injury-prevention and rehabilitation of injured athletes, always focusing on their full recovery and reinjure risk reduction.

\section{PALAVRAS-CHAVE / KEYWORDS}

Râguebi, traumatologia, biomecânica, placagem, concussão cerebral, prevenção Rugby, traumatology, biomechanics, tackle, cerebral concussion, prevention

\section{Introdução}

O râguebi surgiu no ano de 1823 em Inglaterra quando William Webb Ellis, estudante numa escola da localidade de Rugby, Reino Unido, desafiando as regras de um jogo de futebol, pegou na bola com as mãos e correu até à baliza adversária. ${ }^{1}$ Atualmente trata-se de uma das modalidades mais populares no mundo, contando com cerca de 9 milhões de praticantes em 121 países inscritos na World Rugby (entidade reguladora mundial), sendo um dos desportos com maior crescimento em número de atletas nos últimos anos. É tradicionalmente disputado num campo relvado com uma bola oval entre duas equipas de 15 jogadores, apesar de existirem variantes com 7 (Sevens) e 10 jogadores(Tens).2,3

\section{Biomecânica do râguebi}

O râguebi é um desporto de contacto bastante complexo, constituído predominantemente por atividades de baixa intensidade, como marcha, jogging ou corrida, intercaladas com períodos curtos de alta intensidade, como acelerações/desacelerações, sprints, placagens ou atividades estáticas, maioritariamente anaeróbias. ${ }^{4-6} \mathrm{O}$ passe, o hand-off (figura 1-A), o salto para apanhar a bola, o pontapé em corrida, o pontapé aos postes ou o pontapé de ressalto são outras das habilidades integrantes da modalidade. A imagem de marca deste desporto é a placagem (figura 1-B) que ocorre quando o portador da bola é projetado para o chão por um ou mais jogadores da equipa adversária. As atividades estáticas de alta intensidade, como a formação formação dinâmica (maul, figura 1-F) são componentes cruciais do jogo. ${ }^{6}$ Para executar qualquer um destes movimentos o jogador idealmente adota uma posição que lhe permita produzir maior quantidade de força axialmente, fletindo os joelhos e as ancas e alinhando o tronco, cabeça e pescoço, fazendo de seguida extensão das articulações dos membros inferiores e contacto com um dos ombros no adversário.7,8

Apesar de haver quinze posições com nomes e funções distintas, os jogadores podem ser genericamente separados em dois grupos: Avançados e Três-Quartos. Estes têm claras diferenças antropométricas, sendo que os Três-Quartos tendem a ser mais magros e baixos, o que os torna mais rápidos, explosivos e com maior capacidade aeróbia em relação à sua massa corporal. Já os Avançados tendem a ter as características opostas, no entanto, em termos absolutos, produzem mais trabalho global, percorrem maiores distâncias e intervêm em períodos mais longos de trabalho aeróbio e anaeróbio. 6,9 São estes últimos que participam na maioria das atividades estáticas de alta intensidade sendo, por esse facto, mais vantajoso para este grupo possuírem maior capacidade anaeróbia. ${ }^{10}$ A profissionalização do râguebi em 1995 teve como consequência o desenvolvimento da modalidade, o nível de competição aumentou e os atletas tornaram-se mais rápidos e fortes. Isto teve como consequência o aumento da força produzida nos impactos, traduzindo-se desde então no aumento do número de lesões associadas a este desporto. ${ }^{6,11}$

\section{Traumatologia do râguebi}

A combinação da elevada intensidade física com colisões e contactos resulta num risco substancial de lesões neste desporto. De facto, o râguebi é das modalidades coletivas profissionais com uma das mais elevadas taxas de incidência de lesões traumáticas, por exemplo superior à do futebol ou à do hóquei no gelo. ${ }^{12,13}$ Uma importante 
metanálise de Williams et al. sobre lesões no râguebi reportou a incidência durante a competição de 81 lesões por 1000 horas jogador-jogo (HJJ). ${ }^{13}$ Esta é muito superior à verificada durante o período de treino (3 lesões por $1000 \mathrm{HJJ}$ ), sendo maior em equipas profissionais quando comparadas com amadoras (123 versus 35 lesões por 1000 HJJ). Esta última diferença pode ser explicada pelo maior peso e tamanho dos atletas, épocas mais longas, maior nível competitivo, regimes de vigilância de lesões mais eficazes, maior distância percorrida a maiores velocidades e menos tempo de jogo morto verificados no râguebi profissional. 7,14-17

O mecanismo de lesão mais frequente ocorre durante a placagem, mais especificamente ao ser placado. A ação de placar e o ruck/ maul encontram-se em segundo e terceiro lugar, respetivamente. ${ }^{13} \mathrm{~A}$ gravidade das lesões reportadas, definida como o tempo de recuperação da lesão, foi em média de 20 dias. Apesar das lesões de novo ocorrerem mais frequentemente do que lesões recorrentes, estas últimas implicam um tempo de paragem, correspondente em média a mais 10 dias em relação às novas lesões. ${ }^{13}$ Os tipos de lesão mais frequentemente reportados no râguebi são as roturas musculotendinosas e ligamentares. Os membros inferiores destacam-se como o segmento mais afetado, apesar das lesões dos membros superiores, em média, implicarem tempo de paragem superior. ${ }^{13}$ Outras lesões comuns são os traumatismos craniencefálico, fraturas agudas ou de stress nos membros e coluna e lacerações. ${ }^{13}$ A lesão mais frequente, corroborada por vários estudos, é o hematoma da coxa. Já a lesão que implica mais tempo de paragem desportiva é mais frequentemente a rotura do ligamento cruzado anterior (LCA). ${ }^{14,18-20}$ As roturas ligamentares mais comuns são dos ligamentos colaterais dos joelhos, mais frequentemente roturas parciais do ligamento colateral medial. A luxação glenoumeral anterior ocorre com mais frequência no grupo dos Avançados por habitualmente placarem mais. ${ }^{21-23}$ Em termos de roturas musculotendinosas, a mais frequente é dos músculos isquiotibiais, ocorrendo mais frequentemente no grupo dos

Três-Quartos devido ao maior número de sprints que efetuam. ${ }^{24}$ Por sua vez, as fraturas são particularmente frequentes em crianças e jovens, representando 18-27\% das lesões nestas faixas etárias. ${ }^{25} \mathrm{~A}$ cabeça e o pescoço são também das regiões mais afetadas, havendo uma incidência superior face a outros desportos de contacto de concussões cerebrais e de fraturas e luxações da coluna cervical, associadas ou não a lesão medular. Estas últimas são mais frequentemente causadas por um mecanismo de hiperflexão do pescoço, com ou sem componente de rotação, durante o colapso da Formação Ordenada (figura 1-C) em jogadores da primeira linha. ${ }^{23,26} \mathrm{Um}$ estudo prospetivo americano realizado em competições universitárias demonstrou que as lesões do punho e da mão são 9.6 vezes mais frequentes no râguebi do que no futebol americano, sendo mais diagnosticadas luxações das articulações interfalângicas proximais, dedos em martelo e Jersey fingers, fraturas dos metacárpicos e das falanges. ${ }^{27}$ Outro estudo prospetivo,

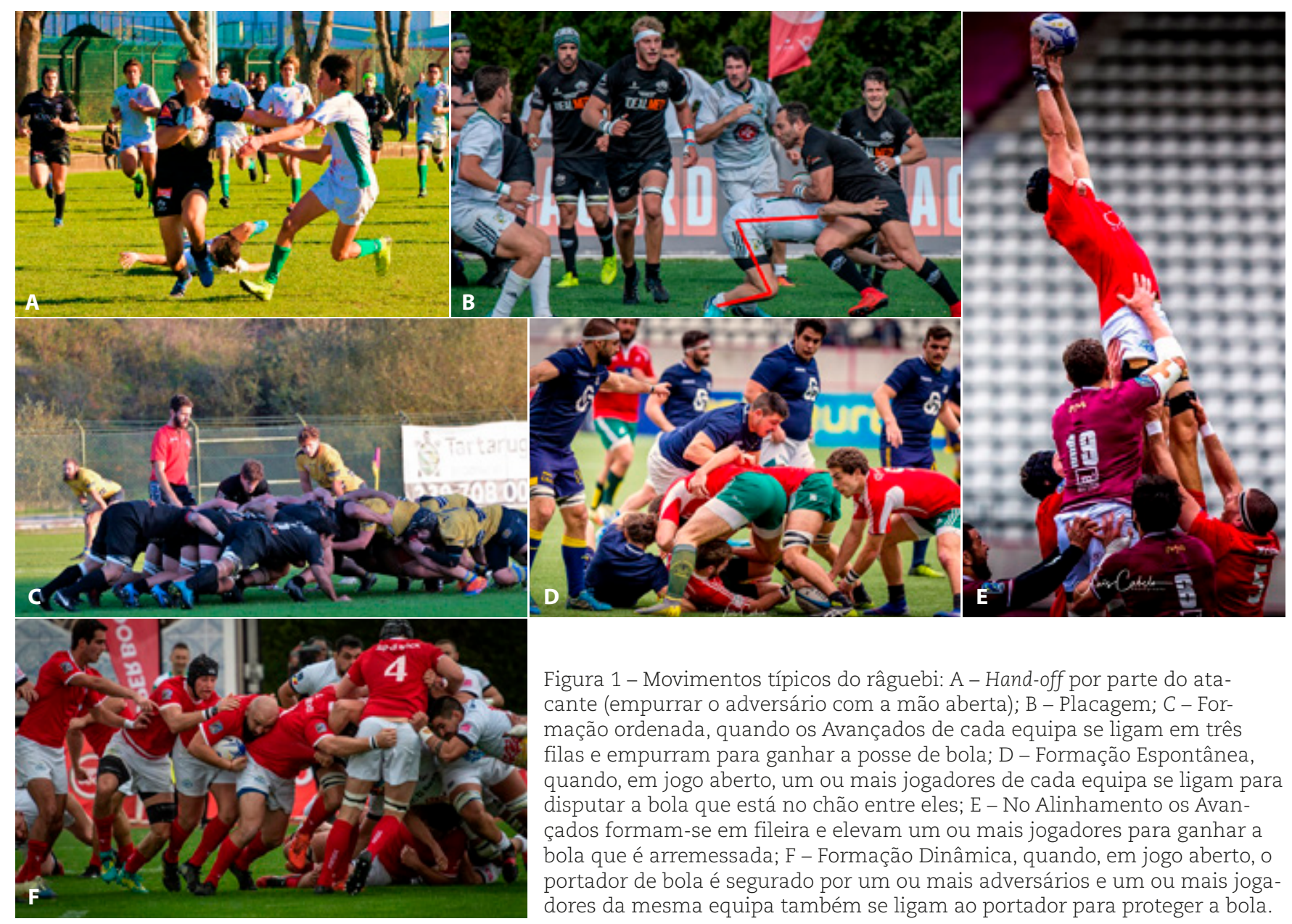


com um coorte de 899 atletas, estabeleceu diferentes padrões de localização de lesões entre Avançados e Três-Quartos. Os primeiros apresentaram mais lesões a nível dos ombros, joelhos e tornozelos ou pés, enquanto os últimos tinham mais incidência de roturas dos músculos isquiotibiais, não se tendo encontrado diferenças no período total de ausência desportiva devido a lesão. ${ }^{28}$ Esse mesmo estudo conseguiu ainda estabelecer diferenças relativas entre cada uma das quinze diferentes posições, referindo-se algumas: jogadores da primeira-linha ( $\mathrm{n}^{\circ} 1,2$ e 3 ) tiveram maiores períodos de paragem devido a lesões da coluna cervical (mais frequentemente hérnias discais, fraturas e luxações), causadas pela pressão a que são sujeitos nas Formações Ordenadas (figura 1-C) e devido às placagens (figura 1-B); os da segunda-linha ( $n^{\circ} 4$ e 5) sofreram relativamente mais de lesões nos tornozelos (entorses, fraturas e luxações) em contexto de quedas durante o Alinhamento (figura 1-E); o $n^{\circ} 6$, abertura $\left(n^{\circ} 10\right)$ e os pontas ( $n^{\circ} 11$ e 14) tiveram mais lesões nas coxas (nomeadamente hematoma e roturas dos músculos isquiotibiais), sobretudo por fazerem um maior número de sprints.

Quanto ao período de jogo, as lesões ocorrem menos no primeiro quarto, sendo que as diferenças de incidências nos outros quartos são menos claras. ${ }^{13}$ No entanto, existe um ligeiro aumento no terceiro quarto (logo após o intervalo). Insuficientes programas de reaquecimento após o intervalo e a redução da concentração mental poderão ser fatores implicados nesta tendência. ${ }^{29}$ Analisando outra variante do jogo, o rugby Sevens (desporto Olímpico desde 2016 e em crescente expansão), apresenta, segundo uma revisão sistemática, uma incidência de lesões superior à variante tradicional com 15 jogadores (102 a 120 lesões por $1000 \mathrm{HJJ}$ ) para o mesmo nível competitivo. ${ }^{30,31}$ Além disso, as lesões são mais graves, possivelmente devido ao facto de os Sevens serem jogadores com maior velocidade (e, consequentemente, com maior transferência de energia nas placagens), haver mais sprints e mais mudanças de direção. Em Portugal, dois recentes estudos prospetivos, que seguiram coortes de atletas das equipas do circuito nacional e universitário de râguebi Sevens, identificaram incidências de lesões também bastante elevadas (134 lesões no circuito nacional e 186 lesões no universitário, por 1000 HJJ) semelhante à reportada a nível internacional para a mesma variante, apesar da severidade média ter sido menor. ${ }^{32,33}$ Os tipos de lesão, os eventos e as localizações foram semelhantes às identificadas na literatura internacional. Num dos estudos foi ainda encontrada uma relação inversa entre o número de horas de treino e a gravidade da lesão. ${ }^{32}$ A lesão que atualmente mais discussão tem originado na comunidade rugbística é a concussão cerebral, devido à potencial relação de causalidade com sequelas a longo prazo, como a encefalopatia crónica traumática e deterioração da função cognitiva precoce. ${ }^{34,35}$ Nas competições de elite do râguebi, um estudo prospetivo recente descreveu uma incidência de concussões de 8.3/1000 HJj no râguebi Sevens e 4.5/1000 HJJ no râguebi tradicional de quinze. ${ }^{36} \mathrm{O}$ tempo de afastamento da competição também foi superior no primeiro (média de 19 vs 10 dias, respetivamente). Qualquer uma das variantes tem incidência de concussões inferior à reportada no futebol americano (18.2/1000 HJJ), mas superior a outros desportos coletivos de contacto como, por exemplo, hóquei no gelo (1.8/1000 HJJ). Apesar da investigação nesta área ter crescido nos últimos anos, a evidência de que antigos jogadores de râguebi expostos a concussões cerebrais repetidas tenham propensão a quadros demenciais precoces é atualmente limitada. ${ }^{37,38}$

\section{No râguebi não existe equipa-}

mento de proteção que seja obrigatório, é apenas aconselhada a utilização de proteção dentária. Não é permitido nenhum material de plástico ou metal. Alguns jogadores usam proteções feitas de almofada fina, como capacetes ou ombreiras. No entanto, a evidência de que estes previnam lesões não é clara. ${ }^{39} \mathrm{~A}$ World Rugby tem apostado em programas de prevenção de lesões, tendo lançado em 2019, a nível global, o programa Activate. ${ }^{40}$ Este é um programa de exercícios simples e dinâmicos para serem efetuados durante os treinos e antes das competições, baseado em evidência e com benefícios demonstrados na prevenção de lesões. ${ }^{41}$ Têm como objetivo melhorar o controlo do movimento dos atletas, ativar e melhorar a sua força e potência muscular. Estão incluídos neste programa exercícios específicos para os músculos da nuca que promovem a estabilidade da coluna cervical, algo importante na dissipação de forças de impacto transmitidas ao cérebro, diminuindo o efeito de chicote que promove a concussão cerebral. ${ }^{42}$ É imperativo que os programas de prevenção sejam individualizados, tendo em conta, não só os antecedentes de lesão de cada um, mas também a sua posição de jogo, visto que cada uma tem diferentes padrões de lesão. ${ }^{28}$ Direcionar os Avançados para a prevenção das lesões da coluna cervical e os Três-Quartos para as lesões da coxa, seria um exemplo. Em alguns países têm sido discutidas abordagens mais radicais nos escalões jovens, por exemplo, a proibição de placagem em competições infantis devido a este ser o evento mais associado a lesões e pelo possível dano das concussões cerebrais em idades tão jovens. Estes tipos de medidas inevitavelmente alteram a natureza do desporto e, portanto, devem primeiro ser fundamentadas com fortes evidências científicas do seu benefício. Um artigo de revisão de 2016 concluiu que, além da evidência limitada, o risco de lesão até aos 15 anos é considerado reduzido e vai aumentando com a idade, de forma similar a outros desportos coletivos e com uma forte correlação com a má técnica de placagem. ${ }^{43}$ Isto poderia significar, então, que este tipo de medidas pode inclusive originar um efeito paradoxal, com maior risco de lesões a longo prazo, visto que os atletas mais jovens, caso continuassem a praticar râguebi na idade adulta, perderiam anos de ensino regular e controlado de uma boa técnica de placagem.

\section{Conclusão}

O râguebi tem uma das mais altas incidências de lesão em desportos 
coletivos, o que se deve a uma combinação entre a alta exigência física e a exposição a contactos e colisões de alta energia. É fundamental conhecer os movimentos típicos do râguebi, bem como das lesões mais frequentemente associadas a cada um deles, de modo a poder elaborar programas eficazes tanto na prevenção de lesões como na reabilitação de atletas, visando ao máximo a proteção da saúde dos praticantes deste desporto.

Os autores declaram ausência de conflitos, assim como a originalidade do manuscrito e a sua não publicação prévia.

\section{Correspondência}

Dr. Diogo Lino Moura

Faculdade de Medicina da Universidade de Coimbra

dflmoura@gmail.com

\section{Bibliografia}

1. Beneke R. Rugby football: Fascinating sport, exceptional history and developments, immense potential for research. Int J Sports Physiol Perform. 2015; 10(6):673. doi:10.1123/ IJSPP.2015-0439

2. World Rugby. World Rugby Year In Review 2017. 2018. https://www.world.rugby/welcome-to-rugby/what-is-rugby.

3. World Rugby. What Is Rugby? https://www. world.rugby/welcome-to-rugby/what-is-rugby. Published 2014.

4. Eaton C, George K. Position specific rehabilitation for rugby union players. Part I: Empirical movement analysis data. Phys Ther Sport. 2006; 7(1):22-29. doi:10.1016/j. ptsp.2005.08.006

5. Austin D, Gabbett T, Jenkins D. Repeated high-intensity exercise in professional rugby union. J Sports Sci. 2011; 29(10):1105-1112. doi:10.1080/02640414.2011.582508

6. Duthie G, Pyne D, Hooper S. Applied Physiology and Game Analysis of Rugby Union. Sport Med. 2003; 33(13):973-991. doi:10.2165/00007256-200333130-00003

7. Quarrie KL, Hopkins WG, Anthony MJ, Gill ND. Positional demands of international rugby union: Evaluation of player actions and movements. J Sci Med Sport. 2013; 16(4):353-359. doi:10.1016/j.jsams.2012.08.005

8. Hendricks S, van Niekerk T, Sin DW, et al. Technical determinants of tackle and ruck performance in International rugby union. J Sports Sci. 2018; 36(5):522-528. doi:10.1080/0264041 4.2017.1322216

9. Cahill N, Lamb K, Worsfold P, Headey R, Murray S. The movement characteristics of English Premiership rugby union players. J Sports Sci. 2013; 31(3):229-237. doi:10.1080/ 02640414.2012 .727456

10. Roberts SP, Trewartha G, Higgitt RJ, El-Abd J, Stokes KA. The physical demands of elite English rugby union. J Sports Sci. 2008; 26(8):825-833. doi:10.1080/02640410801942122
11. Fuller CW, Sheerin K, Targett S. Rugby world cup 2011: International rugby board injury surveillance study. Br J Sports Med. 2013; 47(18):1184-1191. doi:10.1136/bjsports-2012-091155

12. Brooks JHM, Kemp SPT. Recent Trends in Rugby Union Injuries. Clin Sports Med. 2008; 27(1):51-73. doi:10.1016/j.csm.2007.09.001

13. Williams S, Trewartha G, Kemp S, Stokes K. A Meta-Analysis of Injuries in Senior Men's Professional Rugby Union. Sport Med. 2013; 43(10):1043-1055. doi:10.1007/s40279-0130078-1

14. Brooks JHM, Fuller CW, Kemp SPT, Reddin DB. A prospective study of injuries and training amongst the England 2003 Rugby World Cup squad. Br J Sports Med. 2005; 39(5):288-293. doi:10.1136/bjsm.2004.013391

15. Jakoet I, Noakes TD. A high rate of injury during the 1995 Rugby World Cup. S Afr Med J. 1998; 88(1):45-47

16. Targett SG. Injuries in professional Rugby Union. Clin J Sport Med Off J Can Acad Sport Med. 1998; 8(4):280-285. doi:10.1097/00042752-199810000-00005

17. Quarrie KL, Hopkins WG. Changes in player characteristics and match activities in Bledisloe Cup rugby union from 1972 to 2004. J Sports Sci. 2007; 25(8):895-903. doi:10.1080/02640410600944659

18. Brooks JHM, Fuller CW, Kemp SPT, Reddin DB. Epidemiology of injuries in English professional rugby union: Part 1 match injuries. Br J Sports Med. 2005; 39(10):757-766. doi:10.1136/bjsm.2005.018135

19. Dallalana RJ, Brooks JHM, Kemp SPT, Williams AM. The epidemiology of knee injuries in english professional rugby union. Am J Sports Med. 2007; 35(5):818-830. doi:10.1177/0363546506296738

20. Targett SG. Injuries in Professional Rugby Union. Clin J Sport Med. 1998; 8(4):280-5. doi: 10.1097/00042752-199810000-00005

21. Hodhody G, Mackenzie TA, Funk L. Shoulder injuries in adolescent rugby players. Shoulder Elb. 2016; 8(3):159-166. doi:10.1177/1758573216644565

22. Singh VR, Trewartha G, Roberts SP, England M, Stokes KA. Shoulder Injuries in English Community Rugby Union. Int J Sports Med. 2016; 37(8):659-664. doi:10.1055/s-0042-104414

23. A.E. M, W.W. D. Injury trends and prevention in rugby union football. Curr Sports Med Rep. 2010; 9(3):139-143.

24. Brooks JHM, Fuller CW, Kemp SPT, Reddin DB. Incidence, risk, and prevention of hamstring muscle injuries in professional rugby union. Am J Sports Med. 2006; 34(8):12971306. doi:10.1177/0363546505286022

25. McIntosh, AS SKA. Rugby Injuries. In: Maffulli N CD, ed. Epidemiology of Pediatric Sports Injuries: Team Sports. Karger AG, Basel; 2005: 120-139.

26. Shelly MJ, Butler JS, Timlin M, Walsh MG, Poynton AR, O'Byrne JM. Spinal injuries in Irish rugby: A ten-year review. J Bone Jt Surg Ser B. 2006; 88(6):771-775. doi:10.1302/0301$-620 X .88 B 6.17388$

27. Elzinga KE, Chung KC. Finger Injuries in Foo tball and Rugby. Hand Clin. 2017; 33(1):149160. doi:10.1016/j.hcl.2016.08.007

28. Brooks JHM, Kemp SPT. Injury-prevention priorities according to playing position in professional rugby union players. Br J Sports Med. 2011; 45(10):765-775. doi:10.1136/ bjsm.2009.066985

29. Bathgate A, Best JP, Craig G, Jamieson M. A prospective study of injuries to elite Australian rugby union players... including commentary by Wiley JP. Br J Sports Med. 2002; 36(4):265-269.

30. Engebretsen L, Steffen K. Rugby in Rio in 2016! Br J Sports Med. 2010; 44(3):157. doi:10.1136/bjsm.2010.71555

31. Cruz-Ferreira A, Cruz-Ferreira E, Santiago L, Taborda Barata L. Epidemiology of injuries in senior male rugby union sevens: a systematic review. Phys Sportsmed. 2017; 45(1):41-48. doi:10.1080/00913847.2017.1248224

32. Cruz-Ferreira AM, Cruz-Ferreira EM, Silva JD, Ferreira RM, Santiago LM, Taborda-Barata L. Epidemiology of injuries in Portuguese senior male rugby union sevens: a cohort prospective study. Phys Sportsmed. 2018; 46(2):255-261. doi:10.1080/00913847.2018.1441581

33. Silva JD, Ribeiro CAF, Santiago LM. Perfil do jogador de Rugby de 7 Universitário Português: Características Antropométricas e Experiência na Modalidade, bem como da incidência de lesões desportivas. Repositório Científico da UC. 2017.

34. Raftery M. Concussion and chronic traumatic encephalopathy: International Rugby Board's response. Br J Sports Med. 2014; 48(2):79-80. doi:10.1136/bjsports-2013-093051

35. McKee AC, Stein TD, Nowinski CJ, et al. The spectrum of disease in chronic traumatic encephalopathy. Brain. 2013; 136(1):43-64. doi:10.1093/brain/aws307

36. Fuller CW, Taylor A, Raftery M. Epidemiology of concussion in men's elite Rugby-7s (Sevens World Series) and Rugby-15s (Rugby World Cup, Junior World Championship and Rugby Trophy, Pacific Nations Cup and English Premiership). Br J Sports Med. 2015; 49(7):478483. doi:10.1136/bjsports-2013-093381

37. McMillan TM, McSkimming P, Wainman-Lefley J, et al. Long-Term health outcomes after exposure to repeated concussion in elite level: Rugby union players. J Neurol Neurosurg Psychiatry. 2017; 88(6):505-511. doi:10.1136/jnnp-2016-314279

38. Gallo V, McElvenny D, Hobbs C, et al. BRain health and healthy AgeINg in retired rugby union players, the BRAIN Study: Study protocol for an observational study in the UK. BMJ Open. 2017; 7(12):1-12. doi:10.1136/bmjopen-2017-017990

39. McIntosh A, McCrory P, Finch CF. Performance enhanced headgear: A

40. scientific approach to the development of protective headgear. Br J Sports Med. 2004; 38(1):46-49. doi:10.1136/bjsm.2002.003103

41. World Rugby Press Releases. World Rugby launches ground-breaking preventive exercise programme designed to reduce injuries. 09/09/2019. https://www.world.rugby/ news/447540. Published 2019. Accessed January 14, 2020.

42. Hislop MD, Stokes KA, Williams S, et al. Reducing musculoskeletal injury and concus sion risk in schoolboy rugby players with a pre-activity movement control exercise programme: A cluster randomised controlled trial. Br J Sports Med. 2017; 51(15). doi:10.1136/ bjsports-2016-097434

43. Hamilton DF, Gatherer D, Robson J, et al. Comparative cervical profiles of adult 
and under-18 front-row rugby players:

implications for playing policy. BMJ Open.

2014; 4(5):e004975. doi:10.1136/bmjo-

pen-2014-004975

44. Tucker R, Raftery M, Verhagen E. Injury

risk and a tackle ban in youth Rugby Union:

Reviewing the evidence and searching for tar-

geted, effective interventions. A critical review.

Br J Sports Med. 2016; 50(15):921-925.

doi:10.1136/bjsports-2016-096322 